AIAA-98-4869

\title{
STRUCTURAL AND CONTROL SURFACE DESIGN FOR WINGS USING THE ADAPTIVE MODELING LANGUAGE
}

\author{
Jeffrey V. Zweber* \\ Air Vehicles Directorate \\ Air Force Research Laboratory \\ WPAFB, OH 45433-7542
}

\author{
Alicia Hartong $\dagger$ \\ Dept. of Mechanical \& Materials Eng. \\ Wright State University \\ Dayton, OH 45435-0001
}

\begin{abstract}
Computerized engineering environments promise to significantly improve the processes for designing complex mechanical systems. This paper investigates the application of the Adaptive Modeling Language ${ }^{\circledR}$ $\left(\mathrm{AML}^{\mathrm{TM}}\right)$ to the aircraft design process. Models were developed in AML to perform a limited trade study between the sizing of a wing box for high speed maneuvers and the available roll control power at landing speeds. This project has build upon some previous efforts that also used AML. While the results of the trade study were not very interesting, the project did illustrate the advantages of combining diverse disciplines in a single engineering environment. It demonstrated the ease with which existing capabilities in the environment can be reconfigured and applied to a new engineering problem. This project also added to the confidence that a much more expansive system can be developed.
\end{abstract}

\section{INTRODUCTION}

A critical factor in the development of a new product is the time spent on its design. Design time influences both the time it take to get the product to the market, which may significantly affect its success, and the cost to develop the product.

The design of complex mechanical systems is virtually always a team effort. There are simply too many disciplines needed for one engineer to master and too much work to be done in a timely fashion. Two factors impact how efficiently and quickly the design team can work together, their ability to communicate and the time needed for each engineering task. Good communication is needed to ensure that each team member has access to the information he needs and that the information is upto-date. The time for an individual engineering task is

*Aerospace Engineer, AIAA Member

$\dagger$ Research Engineer

This paper is declared work of the U.S. Government and is not subject to copyright protection in the United States. important because another designer may be delayed if he is waiting for the results. If the goal is to shorten the design time, shortening the time of the individual tasks seems like a good place to start.

The US defense industry has recognized that the design of modern military aircraft share these characteristics with the design of other complex mechanical systems. The concerns outlined above are becoming ever more important for the designers of new military aircraft because they are required to consider an increasing number of disciplines (e.g., survivability, cost, supportability) earlier in the design process. This requirement is driving up the number of engineers that need to be included on the conceptual and preliminary-level design teams. Consequently, this increases the importance of good communication and the rapid turnaround of engineering analyses.

In hopes of addressing these concerns, all the major US airframers, the Department of Defense and NASA are working with engineering environments. Four engineering environments that are currently being used and improved are: Simulation Based Design[1], which is being developed by Lockheed Martin Missiles \& Space; TechnoSoft's Adaptive Modeling Language[2]; IMAGE[3], developed by Georgia Tech's Aerospace Systems Design Laboratory; and Engineous Software's iSIGHT[4]. (Note: this list was not intended to be extensive, only to give the reader a few examples of engineering environments.)

The MultiDisciplinary Design Integrated Product Team (MDDIPT) of the Air Force Research Laboratory's Air Vehicles Directorate has been working with the Adaptive Modeling Language (AML) since the summer of 1996. Thus far, the IPT has focused on three areas of research: the conceptual design process, the preliminary design process and some tools needed for design. In the area of conceptual design, the research that is being done is focused on being able to build models rapidly. These models also need to capture the toplevel effects of design decisions[5]. The research efforts are seeking to develop capabilities that enable the designer who is developing the aircraft's configuration to also work with both operations researchers and the 
engineers who are working on more detailed design decisions.

At the preliminary level of design, the IPT is working on the capability to access various disciplines from a single design environment[6]. Besides simply accessing various preliminary-level design tools, the research has examined the benefits of automating the repetitive engineering tasks that occur at this level of design. This paper documents some of the research performed in this area.

The general design tools research has concentrated on automatic differentiation and optimization[7]. This research is needed to examine the proper place for optimization in the overall design process. Optimization has been shown to be very useful in specific disciplines, but many designers are resistant to the idea that a global optimization procedure can be developed that will encompass every discipline involved in the design of an aircraft.

On the basis its two years of experience with AML, and its examination of other engineering environments and multidisciplinary design efforts, the MDDIPT is working on the development of the Aerospace Technology Assessment System (ATAS)[8]. As a first step, the IPT has developed a list of requirements for the engineering environment in which ATAS will be developed. The IPT has not uncovered an environment that meets all of these requirements, but the IPT believes they are feasible because it has identified at least one environment that already meets each requirement individually.

The research described in this paper can form the basis for some of the analysis capabilities that will be required for ATAS. This effort was intended to serve as a proof-of-concept for the integration of various diverse preliminary-level design tools in a single engineering environment.

\section{METHODOLOGY}

This project has built upon the initial structural and manufacturing modeling work that was performed in the AML environment[6]. In this previous work, methods were developed for defining the outer moldline surface of a wing, generating a preliminary-level finite element model of a wing and using an activity-based cost model to determine the manufacturing cost of a wing. For this project, the outer surface models were used directly from the previous work. In addition, the finite element modeling capability was extended and an expanded aerodynamic modeling capability was developed.

\section{Surface Modeling of the Wing}

The first step in this wing design process is to specify the outer moldline. The procedure used for this project is identical to the one used for the previous effort. First, a planform and an airfoil section are defined. To this planform, dihedral and twist are added to position the airfoils in space. (Note: the user has control over the number and position of these airfoils on the twisted planform.) The airfoils are then "skinned" to create the outer moldline surface model.

AML is also capable of importing the outer moldline surface via standard geometry file formats (i.e., IGES, STEP). To use imported surfaces with this project, methods will need to be developed to calculate the planform parameters from the imported geometry. Similar "geometric reasoning" capabilities have been developed for other AML applications.

After the planform and outer moldline surface are modeled, a 2-D (surface) finite element model (FEM) and panel aerodynamic model can be generated. Because this project takes advantage of AML's adaptive class structure and demand driven calculations, the order in which these are created is independent. The only requirement is that both models be developed before the structural optimization can be performed. The FEM and aerodynamic models are covered in the following two sections.

\section{Finite Element Modeling of the Wing}

The objects that are used to generate the finite element mesh for this project are the same as the ones used in the previous effort. The first step in generating the FEM is to layout the substructure (i.e., spars and ribs). This layout is performed on a planform view of the wing. First, a network of points is calculated from the planform outline. The substructure elements are then drawn between these points. Because the points are associated with the planform, when the planform is changed (e.g., new sweep angle) the locations of the substructure are updated as well. A sample of the substructure layout interface is shown in Figure 1.

After the spars and ribs are positioned in the planform view, the models of the surfaces that make up the wing box (i.e., spars, ribs, and upper and lower wing skins) needed to be created. A sample wing box surface model is presented in Figure 2. This model was created using standard geometric calculations (e.g., projections, trim, skin surface) that should be available in any commercial geometry engine or CAD package.

Once the surfaces were generated, the finite element mesh could be created. For this project, AML's bundled routines were used to generate an unstructured tetrahedral mesh. A sample wing box mesh is shown in Figure 3. (Note: the upper surface is not shown to improve the clarity of the figure.)

One of AML's key time-saving features is its ability to capture the strategy behind an engineering activity. This feature was used to generate the complete FEM for 


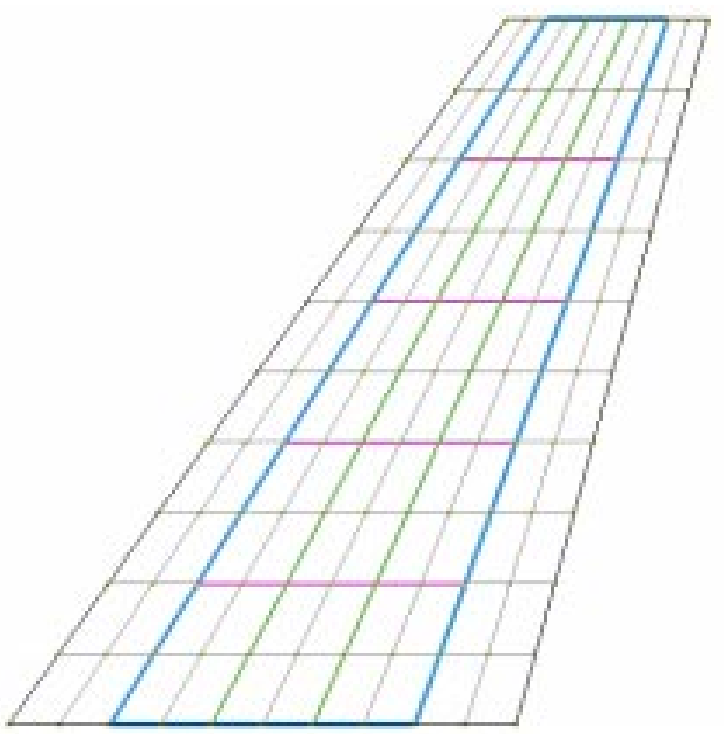

Figure 1: Wing Substructural Layout Interface

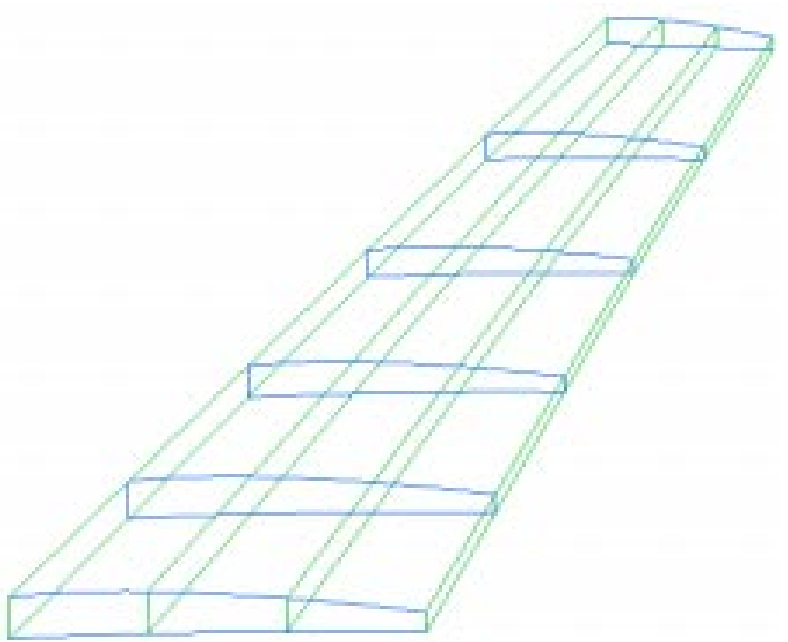

Figure 2: Wing Box Surface Model

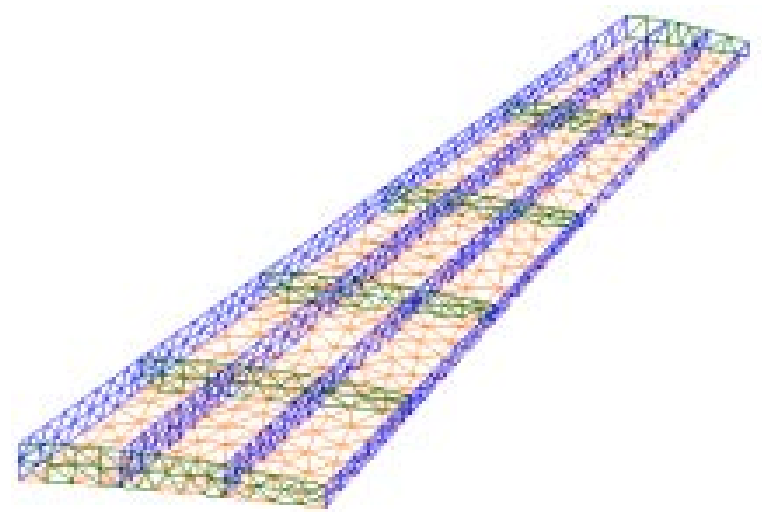

Figure 3: Wing Box Surface Mesh this project. The time savings are the most apparent when the new mesh is needed due to a change in a planform parameter (e.g., a derivative design that is created by just changing the sweep angle). To discover why, we need to examine all the information that is needed for a finite element analysis.

For an analysis, the FEM requires both the mesh and the structural properties (e.g., material properties, thicknesses) of each surface. A shortcoming of typical FEM preprocessors is that they only associate this information with the surface model. The engineer needs to pick each surface and specify the associated information. In this project's scenario, if the planform was changed, the wing box surfaces would need to be recreated. Using the typical FEM preprocessor, the attributes would have been lost when the surface was destroyed.

By capturing the model generation strategy, AML can propagate the necessary information throughout the process. In this effort, the attributes for the FEM were associated with the structures when they were layed-out (Figure 1). These parameters were then passed along to the wing box surfaces (Figure 2) when they were created and finally to the FEM objects.

\section{Aerodynamic Modeling of the Wing}

Along with the finite element model, an aerodynamic model of the wing was required for this project. The model that was developed for this project is for a flat plate aerodynamic analysis.

The layout of the aerodynamic regions is like the way the substructure was layed-out in the previous section. Again, the first step is to define a network of points for the planform. This network may be the same or a separate instance of the object that was used for the substructure. The reason for a separate instance may be to simplify the modeling for the engineer. The designer may not want the divisions in the aerodynamic model to exactly line up with the substructure. For instance, the structural and aerodynamic modeling of a flap may not have the identical geometry.

Once the planform grid is instantiated, the chordwise and spanwise regions can be defined. A model representative of the ones used for this project is shown in Figure 4. This model contains six regions; they were created by two chordwise and one spanwise division.

The aerodynamic analysis chosen for this project requires that the inboard edge of the aerodynamic model coincide with the aircraft centerline. The object developed for this project will add a chordwise region to the model if the associated planform does not extend to the centerline. This was the case for the model shown in Figure 4. The original planform model is only covered by the four outboardmost regions. These regions were 


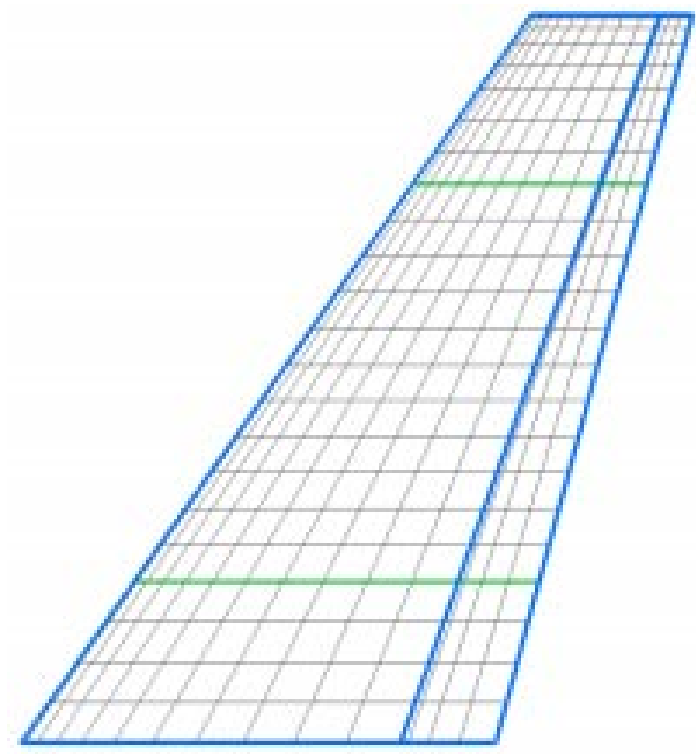

Figure 4: Wing Aerodynamic Model (For Flat Panel Aerodynamic Analysis)

generated by adding one spanwise and one chordwise division to represent an outboard aileron.

After the regions have been defined, the engineer needs to specify values of the parameters used to control the aerodynamic mesh. These parameters control the number of aerodynamic boxes and their distribution within each region (e.g., grouped toward the leading edge, grouped outboard). Because continuity is required between the regions, the mesh attributes are only specified once for each chordwise set of regions (in this case, 3 ) and once for each spanwise set (in this case, 2).

As with the FEM generating procedure, the strategy for generating this model is captured in AML. Consequently, if the planform is changed, the regions will be updated and then the aerodynamic mesh will be recreated. The new mesh will have the same number of boxes in each region and maintain the specified distribution relative to the regional boundaries.

\section{Structural Optimization of the Wing}

Up to this point, the objects for the finite element model and the aerodynamic model are general. These models can be used with a wide range of analysis codes. For this project, additional objects and methods were created specifically to be used with the structural optimization code ASTROS.

ASTROS[9] is designed to determine the optimum thicknesses and cross-sectional areas of finite elements, subject to a wide variety of constraints, loading conditions and objective functions. AML objects were developed to model some of these constraints and loading conditions; however, only the default, minimum weight, objective function was supported. Additionally, proper- ties were added to the AML FEM objects to identify which elements were to be designed and to specify side constraints on their thicknesses or cross-sectional areas.

The two constraints used for this project were the von Mises stress constraint and the aileron effectiveness constraint. The objects for the von Mises constraint simply associated the maximum stress values with specific elements or material properties. The aileron effectiveness constraint object was used to link a constraint value to the aerodynamic model of a control surface.

The loading conditions used for this effort were the aerodynamic forces generated by two maneuvers. ASTROS uses a splining technique to transfer the loads from the aerodynamic model to the structural model. An AML object was developed to model the input requirements for this spline. Besides the spline, ASTROS requires information about the maneuver to perform the aerodynamic calculations. Objects were developed to support two types of steady maneuvers, symmetric (e.g., pull-up) and antisymmetric (e.g., roll). For each of these maneuvers, ASTROS requires the flight condition information (i.e., Mach number, dynamic pressure) as well as free-body rates, accelerations and the deflections of the control surfaces.

\section{Determination of Stability Derivatives for the Wing}

The structural optimization of a wing for aeroelastic maneuvers is a well-known problem that can be, and has been, solved by ASTROS[10]. Simply putting a more user-friendly interface on the front end of ASTROS does not contribute much to the improvement of this multidisciplinary design process. For this project, it was desired to demonstrate a design process that includes a consideration beyond those normally used in an ASTROS optimization. The design consideration that was chosen was the roll control power at landing.

Typically, the landing roll control power is an input requirement that is used to determined the size of the control surfaces. This is because at landing speeds, the flexibility (aeroelastic) effects on the wing are small. By adding a landing roll power requirement, this project has devised a multidisciplinary design problem that must consider both control surface sizing and aeroelastic structural performance at the same time.

While there are many aerodynamic codes that can be used to determine roll control power, this project used ASTROS because of the interface to AML that was already developed. Because of the aeroelastic trim solution routine that ASTROS uses, the program needs to calculate the stability derivatives for each control surface. (Note: the roll control power can be calculated from the aileron stability derivative by using the appropriate flight condition and aircraft geometric parameters.) 
Although the desired stability derivative is calculated by ASTROS during an antisymmetric aeroelastic trim analysis (and even printed in the output), it is not stored in the database that is created by the standard version of the program. This feature was added to the AANDE version of ASTROS[11]. However, because this project uses the standard version of the program, another method was found to retrieve the stability derivative information from ASTROS. It was discovered that ASTROS provides a general stability derivative constraint formulation. By setting the appropriate values for this constraint, the nondimensionalized constraint value, which is written to the database, could be made to be the stability derivative of interest.

Once the information was in the ASTROS database, it was a relatively straightforward process to transfer the information to AML. A general FORTRAN program was written to read a relation name and description from an ASCII file, retrieve a relation from the ASTROS database and store the data in a separate ASCII file. A method was then developed in AML to generate the input ASCII file, call the FORTRAN program and read the information from the second ASCII file into AML.

\section{Cost Modeling}

Although they were not required for this project, the activity-based cost (ABC) models that were developed for the previous effort could have been easily incorporated into this model. This possibility is enabled by the fact that AML is an object-oriented programming (OOP) language. In the previous project, the ABC models were developed as objects. These objects only require a link to the wing box object to calculate the cost of manufacturing the wing box. The $\mathrm{ABC}$ model objects contain the information on the manufacturing process and they know how to extract the other information they need (i.e., geometric data) from the wing box object.

In addition to using OOP, AML's adaptive class structure makes it easy to add the ABC models to this project at any time. What the adaptive class structure provides is the ability to add objects to a previously instantiated model. For instance, if the engineer using this project had already built the FEM and run ASTROS, and then decided to determine the cost of his design, he could add the ABC objects to the existing model hierarchy and establish the links to the wing box objects. This flexibility allows the designer to work on parts of the model without having to consider all the possible analyses he may need beforehand. The engineer is allowed to build the model as the design progresses. There is no need recreate the object hierarchy, and possibly redo work, with each additional discipline that is used in the design process.

\section{Postprocessing}

Many times during the use of ASTROS, and other complex engineering analyses, the designer wants to look at more information than just the final result. In the case of an ASTROS optimization for an aeroelastic problem, the engineer is often interested in the aerodynamic pressures that were calculated by USSAERO. This information is useful, for instance, to verify that the number and distribution of the aerodynamic boxes is reasonable.

While it is possible to visualize the aerodynamic pressures in many engineering utilities, it seems the most convenient to analyze the results in the same environment that the model was built. This data management capability, and the simplification for the designer, is, after all, the promise of modern engineering environments. Following this reasoning, objects and methods were developed for this project to retrieve the aerodynamic data from the ASTROS database and display it as a fringe plot. A sample plot is shown in Figure 5. It

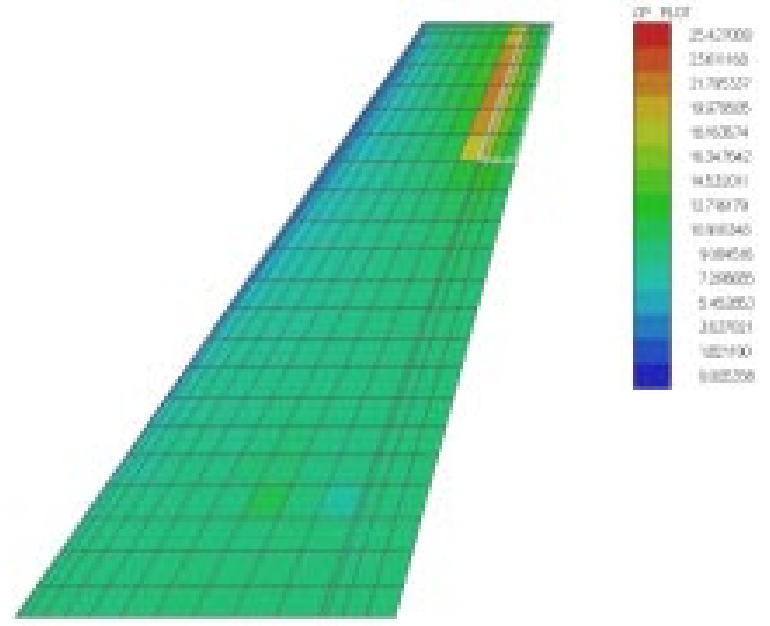

Figure 5: Aerodynamic Pressure Distribution for the Wing

should be noted that the object for creating the fringe plot is general and can be used with data that is not generated by ASTROS.

\section{RESULTS}

The models, objects and methods described above were integrated into a single AML application. This application was designed to allow an engineer to perform some preliminary-level development studies for a wing from within a single integrated environment. Because of the capabilities of the individual objects, the overall application allows the designer to vary the wing outer moldline shape (both airfoil section and planform parameters), the substructural arrangement (i.e., the 
number and placement of the spars and ribs) and the size (and number) of control surfaces.

\section{An Example Trade Study}

The application was validated by performing a sample trade study. The validation study assumed that the design required a specified planform shape and an outboard aileron. The values of the planform parameters that were used are given in Table 1 . The wing character-

Table 1: Planform Parameters

\begin{tabular}{|l|l|}
\hline Span & $140 \mathrm{in.}$ \\
\hline Root Chord & $100 \mathrm{in}$. \\
\hline Taper Ratio & 0.4 \\
\hline Sweep of Leading Edge & $35^{\circ}$ \\
\hline Dihedral & $0^{\circ}$ \\
\hline Twist & $0^{\circ}$ \\
\hline
\end{tabular}

istics that were varied in the study are: the airfoil section, the aileron's span length, and the substructural arrangement. It should be noted that the aileron's chord length was defined as the distance from the trailing edge spar to the trailing edge of the wing surface. Therefore, varying the substructural arrangement can affect the aileron's chord length. The values of the parameters used for this study are given in Table 2 .

The AML models were used to generate 12 ASTROS finite element models, each with a different combination of the trade study parameters. ASTROS was then used to design the wing box for two aeroelastic maneuvers. The wing box was modeled as an aluminum structure; with the wing skins (top and bottom), spars and ribs (except for the root rib) being sized individually to determine the minimum weight design. For the configuration shown in Figure 2, this formulation results in 11 design variables.

The loading conditions used in the study were the aerodynamic forces generated by a $3 \mathrm{~g}$ symmetric pullup and a steady roll. Both maneuvers were modeled at $\mathrm{M}=0.9$ at sea level. The constraints used in the problem were material stresses for both flight conditions and the aileron effectiveness for the steady roll maneuver.

Once the minimum weight wing box was determined for each condition, the flexible stability derivative for the aileron was calculated. This stability derivative was calculated for a roll maneuver at $140 \mathrm{kts}$. at sea level. This flight condition and maneuver was chosen because the resulting stability derivative can be used as a metric of the roll control power available at landing.
Table 2: Trade Study Geometric Parameters

\begin{tabular}{|c|c|}
\hline \multirow{2}{*}{ Airfoil Sections } & 4408 \\
\hline & 4406 \\
\hline \multirow{3}{*}{$\begin{array}{l}\text { Aileron Span } \\
\text { Note: chord is } \\
\text { defined by TE spar } \\
\text { location }\end{array}$} & $30 \%$ of the wing span \\
\hline & $35 \%$ of the wing span \\
\hline & $40 \%$ of the wing span \\
\hline \multirow{2}{*}{$\begin{array}{l}\text { Substructural } \\
\text { Arrangements }\end{array}$} & $\begin{array}{l}\text { Wing Box LE Spar @ 20\% } \\
\text { Wing Box TE Spar @ 80\% } \\
\text { Two Intermediate Spars @ } \\
40 \% \text { and 60\% } \\
\text { Four Intermediate Ribs = } \\
20 \%, 40 \%, 60 \% \text { and } \\
80 \%\end{array}$ \\
\hline & $\begin{array}{l}\text { Wing Box LE Spar @ 20\% } \\
\text { Wing Box TE Spar @ 74\% } \\
\text { Two Intermediate Spars @ } \\
38 \% \text { and 56\% } \\
\text { Four Intermediate Ribs = } \\
20 \%, 40 \%, 60 \% \text { and } \\
80 \%\end{array}$ \\
\hline
\end{tabular}

The purpose of this example trade study is to compare the study parameters (Table 2) effect on the optimum weight of the wing box and the landing control power. A good design is one that minimizes the weight of the wing box while maximizing (or meeting a threshold value of) the landing control power. The optimized weights and control powers are shown in Figure 6 for all 12 cases. (Note: in the legend for Figure 6, S-1 corre-

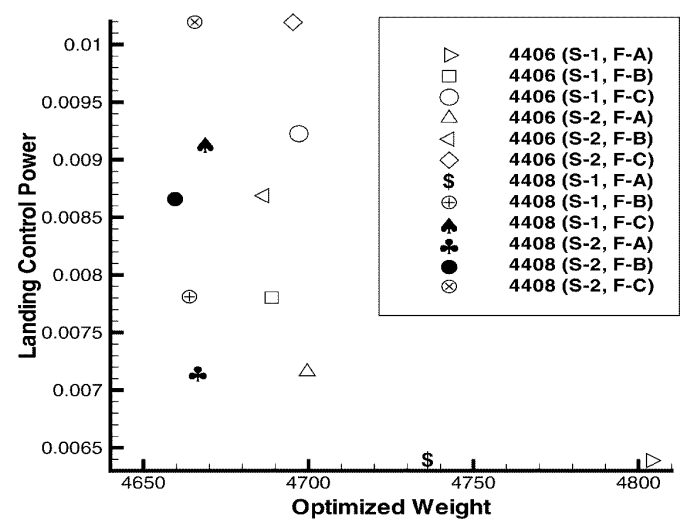

Figure 6: Trade Study Results

(Landing Control Power vs. Optimized Weight)

sponds to the structure with the $80 \%$ trailing edge spar and $\mathrm{S}-2$ represents the structure with the $74 \%$ trailing 
edge spar. Also, F-A, F-B and F-C correspond to the ailerons with $30 \%, 35 \%$ and $40 \%$ spans, respectively.)

A few predictable observations can be made from Figure 6. First, with all other things been equal, the thinner wing box is heavier. This can be seen by noting that the 4406 cases are each heavier than the corresponding design using the 4408 airfoil section. Second, the larger ailerons generate more control power than the smaller ones. This conclusion is obvious for a rigid wing, but not necessarily true for a flexible one. For a flexible wing, conventional ailerons generate a twisting moment that deforms the wing in such a way that it counteracts the desired change in lift on each side of the aircraft. However, for this trade study, the landing speeds are slow enough that this effect is minor.

An interesting observation about Figure 6 is that the optimized weights seem to correspond very well to the airfoil section, except for the cases with the smallest aileron (S-1, F-A). This result is caused by a difference in the constraints that drove the optimization. The constraints were the same for all cases; however, in the cases with the smallest aileron, the aileron reversal constraint was most important one in the design. In the other cases, the design was driven by the von Mises stress constraints for the $3 \mathrm{~g}$ symmetric pull-up maneuver.

Overall, the results of this trade study are not that exciting. The weight of the wing box is only driven by the airfoil section as long as a minimum aileron size is chosen. Also, a wide range of landing control powers can be achieved by varying the size of the aileron. However, the purpose of this project was not to design a wing box; it was to demonstrate the advantages of developing a complex design process in a single engineering environment.

\section{CONCLUSIONS}

This project has demonstrated the integration of preliminary-level structural and control surface sizing analysis in a single engineering environment. It has shown that the results of preliminary-level engineering analyses can be used to rapidly determine the effects of conceptual-level design decisions. Together with the previous efforts of the MDDIPT, this effort has shown that significant improvements to the design process are possible and that the development of ATAS is feasible.

\section{REFERENCES}

1 Simulation Based Design web site, http:// sbdhost.parl.com/
2 "The Adaptive Modeling Language. A Technical Perspective", TechnoSoft, Inc., Cincinnati, OH, 1998.

3 IMAGE web site, http://alpha.cad.gatech.edu/ image/index.html

4 Engineous Software, Inc. web site, http:// www.engineous.com/

5 Blair, M., Hill, S., Crossley, W., Taylor, R. and Weisshaar, T. A., "Rapid Modeling with Innovative Structural Concepts," AIAA paper 98-1755, presented at the 39th AIAA/ASME/ASCE/AHS/ASC Structures, Structural Dynamics and Materials Conference, April 20-22, 1998.

6 Zweber, J. V., Blair, M., Kamhawi, H., Bharatram, G. and Hartong, A., "Structural and Manufacturing Analysis of a Wing Using the Adaptive Modeling Language", AIAA paper 98-1758, presented at the 39th AIAA/ASME/ASCE/AHS/ASC Structures, Structural Dynamics and Materials Conference, Long Beach, CA, April 20-23, 1998.

7 Veley, D. E., "Optimization in the Adaptive Modeling Language", AIAA paper $98-4872$, to be presented at the 7th AIAA/USAF/NASA/ISSMO Symposium on Multidisciplinary Analysis and Optimization, Sept. 2-4, 1998.

8 Veley, D. E., Blair, M., and Zweber, J. V., "Aerospace Technology Assessment System", AIAA paper 98-4825, to be presented at the 7th AIAA/ USAF/NASA/ISSMO Symposium on Multidisciplinary Analysis and Optimization, Sept. 2-4, 1998.

9 Neill, D. J. and Herendeen D. L., ASTROS Enhancements, "Vol. I: ASTROS Users Manual", WL-TR-96-3004, "Vol. II: ASTROS Programmers Manual", WL-TR-96-3005, "Vol. III: ASTROS Theoretical Manual", WL-TR-96-3006.

10 Love, M. H., Barker, D. K., and Bohlmann, J. D., "An Aircraft Design Application Using ASTROS", WL-TR-93-3037.

11 Zink, P. S., Mavris, D. N., Love, M. H., Karpel, M. "Robust Design for Aeroelastically Tailored/Active Aeroelastic Wing", AIAA paper 98-4781, to be presented at the 7th AIAA/USAF/NASA/ISSMO Symposium on Multidisciplinary Analysis and Optimization, Sept. 2-4, 1998. 